\title{
Kompetenz in allen Bereichen
}

Das Interview führte: Matthias Scholer

Die ärztliche Aus-, Weiter- und Fortbildung ist in Bewegung. Welche ambitiösen Ziele das SIWF in den kommenden zehn Jahren verfolgt, erläutert die oberste Verantwortliche für die ärztliche Weiter- und Fortbildung in der Schweiz, Monika Brodmann Maeder. Sicher ist, dass es in allen Bereichen zu grundlegenden Änderungen kommen muss, wenn wir die Arbeitsqualität unserer Ärztinnen und Ärzte auch künftig auf hohem Niveau halten wollen.

\section{Frau Brodmann, Sie leiten seit diesem Februar das} SIWF. Welche Zwischenbilanz können Sie ziehen? Ich habe ein professionelles und höchst motiviertes Team von meinem Vorgänger übernommen. Dank diesem kann ich mich effizient um die anstehenden Reformen im Bereich Weiter- und Fortbildung kümmern.

Lassen Sie uns zuerst über die anstehenden Veränderungen in der Weiterbildung sprechen. Worauf legen Sie in diesem Bereich den Fokus?

Auf die Einführung der kompetenzbasierten Weiterbildung. Mein Vorgänger Werner Bauer hat in diesem Bereich schon wertvolle Vorarbeit geleistet. Mein Ziel ist es nun, die CBME (Anmerk. d. Redaktion CBME - Competency-Based Medical Education) über alle Fachgebiete hinweg, für sämtliche Ärztinnen und Ärzte in Weiterbildung und in jeder Weiterbildungsstätte zu implementieren.

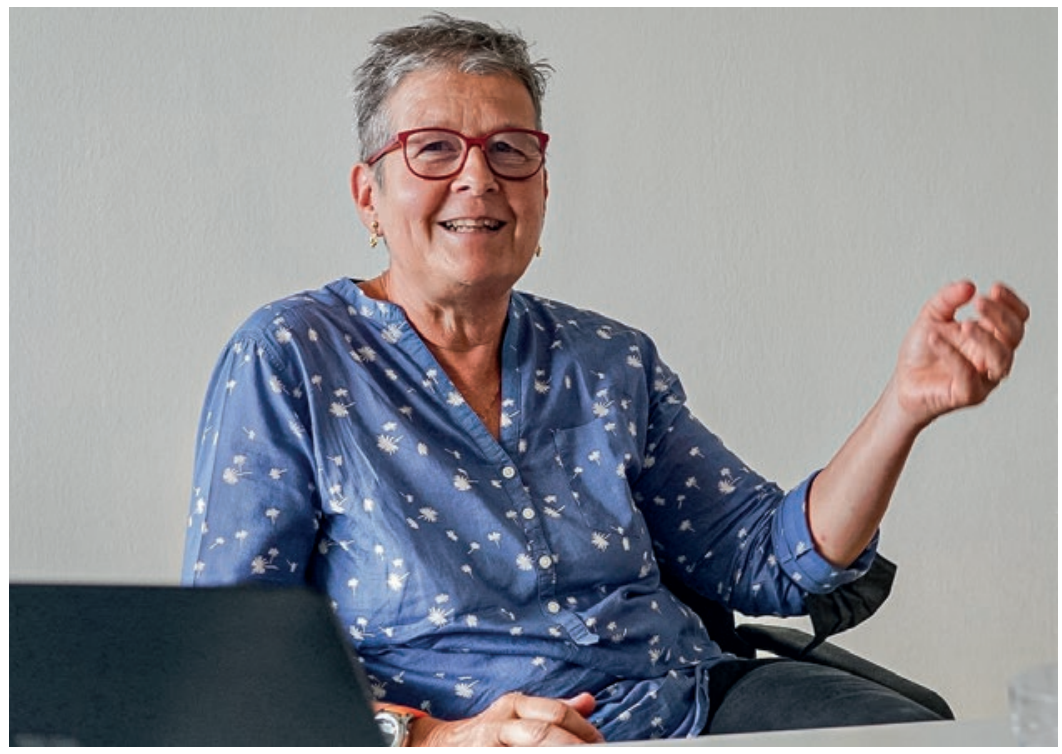

Monika Brodmann will der kompetenzbasierten Weiterbildung zum Durchbruch verhelfen.
CBME ist seit den 1970er-Jahren immer wieder ein Thema. Obwohl der kompetenzbasierte Ansatz logisch und zielführend scheint, konnte sich diese Form der Weiterbildung in der Schweiz bislang kaum durchsetzen. Weshalb?

Dafür gibt es verschiedene Gründe. Ein entscheidender Punkt ist sicherlich, dass in der universitären Ausbildung der Medizinstudierenden die Mehrheit der Professorinnen und Professoren ihrerseits mittels klassischen Methoden wie Frontalunterricht und einem grossen Anteil an Selbststudium unterrichtet wurden. Diese Unterrichtsform haben sie dann später in ihrer Rolle als Aus- und Weiterbildende übernommen. Zudem verläuft bis heute die typische Laufbahn zu einer Professur über Forschung, Publikationen und eine Habilitation im jeweiligen Fachbereich. Nur am Rande müssen sich die Anwärterinnen und Anwärter im Rahmen eines Didaktik-Kurses auch mit dem Thema Bildung auseinandersetzen.

\section{Sie plädieren also für eine vertiefte Ausbildung der Weiterbildenden im Bereich der Lehre?}

Ja, in einem ersten Schritt vor allem für Personen in leitenden Positionen. Wir dürfen nicht davon ausgehen, dass eine gute Ärztin oder ein guter Forscher automatisch auch eine gute Lehrperson ist - "teachers are made, not born». Wir sagen auch nicht, eine Lehrerin, ein Lehrer kann ohne entsprechende Ausbildung an den Schulen Wissen vermitteln. Auch sie müssen eine entsprechende Ausbildung durchlaufen und sich danach kontinuierlich weiter- und fortbilden.

Sie haben an der Universität Bern einen Master in Medical Education absolviert. Welche Erfahrungen machten Sie dabei?

Mir eröffnete sich eine neue Welt. Ich war immer der Meinung, dass ich in meinen medizinischen Tätigkei- 
ten mein Wissen ziemlich gut weitergebe. Dank dem Kurs erfuhr ich erst, welche Struktur und auch welches Weltbild hinter der medizinischen Bildung steckt. Ich realisierte, dass Bildung eine eigene Kompetenz ist, die man erlernen und sich aneignen muss. Vor allem in übergeordneten Funktionen, in denen es auch darum geht, Lehrpläne auszuarbeiten oder längerfristige Projekte zu entwickeln, braucht es viel Know-how. Wir brauchen an den entscheidenden Stellen Leute mit Bildungsexpertise und nicht bloss Bildungsbegabung.

Dank der Einführung des Lernzielkataloges PROFILES sollten im Medizinstudium die Curricula hin zu einer kompetenzbasierten Medizin angepasst werden. Wie sind in diesem Bereich die Fortschritte?

Grundsätzlich sind mit PROFILES die nötigen Rahmenbedingungen gegeben. Es zeigt sich, dass Fakultäten, die erst seit Kurzem ein Medizinstudium anbieten, es leichter haben, ein neues Curriculum zu entwickeln und im

Wir brauchen an den entscheidenden Stellen Leute mit Bildungsexpertise und nicht bloss Bildungsbegabung.

Alltag zu implementieren. Zu den Universitäten, die neu dazugestossen sind und ihre Studiengänge quasi auf der grünen Wiese entwickeln konnten, gehören die Universitäten St.Gallen, Fribourg und Lugano und die ETH Zürich.

Gibt es ähnliche Vorreiter seitens der Fachgesellschaften? Ein Musterbeispiel ist die Kardiologie. Die Kardiologen haben ihr Weiterbildungsprogramm komplett kompetenzbasiert ausgerichtet. Der Vorstand des SIWF hat dieses bereits verabschiedet. Bis zur Umsetzung müssen wir nun jedoch noch abwarten, bis alle beteiligten Weiterbildungsstätten entsprechend vorbereitet sind, die Weiterzubildenden entsprechend betreuen zu können. Auch das Swiss College of Surgeons ist dabei, ein kompetenzbasiertes Konzept für ihre Ärztinnen und Ärzte in Weiterbildung einzuführen. Vonseiten Alterspsychiatrie, Gynäkologie und Geburtshilfe und weiteren Fachgesellschaften wird ebenfalls Interesse an einer entsprechenden Überarbeitung ihrer Weiterbildungsprogramme signalisiert. Das SIWF unterstützt sie dabei.

Im Vergleich zu den herkömmlichen Programmen, die über zwanzig Seiten bis ins letzte Detail aufzählen, was man während einer Weiterbildung darf und was nicht, kommen die kompetenzbasierten Programme viel einfacher und klarer rüber. Aber: Mit der Ausarbeitung von EPAs (Anmerk. der Redaktion: EPA - Entrustable Professional Activities) allein ist es nicht getan. Es braucht flankierend einen Kulturwandel aller beteiligten Player auf sämtlichen Stufen.

\section{Wie kann bei den Weiterbildungsstätten ein entspre-} chender Kulturwandel angestossen werden?

Erstens müssen wir uns zusammen mit den Kantonen überlegen, ob wir künftig Weiterbildungsstätten, welche ihre Weiterbildungen kompetenzbasiert anbieten, mit entsprechenden finanziellen Anreizen belohnen sollten. Zweitens müssen wir die Bildungsexpertise bei den Weiterbildnerinnen und Weiterbildern erhöhen Die "teach-the-teacher»-Kurse, welche das SIWF bereits seit einigen Jahren erfolgreich anbietet, sind eine Möglichkeit, sich im Bereich der medizinischen Weiterbildung weiterzuentwickeln. Diese in Zusammenarbeit mit dem Royal College of Physicians of London angebotenen Workshops werden derzeit durch unsere eigenen Bildungsexpertinnen und -experten noch stärker an die Schweizer Verhältnisse angepasst. Zudem wird das Thema der digitalen Möglichkeiten im Weiterbildungsbereich stärker gewichtet.

\section{Wie sieht die Zusammenarbeit mit dem Bund aus?}

Das SIWF muss sich alle sieben Jahre vom Bund als verantwortliche Organisation für die ärztliche Weiterund Fortbildung akkreditieren lassen. Das Bundesamt für Gesundheit, das SIWF und weitere Organisationen haben vor Kurzem eine Begleitgruppe gebildet, um dafür zu sorgen, dass die Akkreditierung der einzelnen Weiterbildungsprogramme vereinfacht wird. Der Akkreditierungsprozess soll nicht als Schikane aufgefasst werden, sondern vielmehr als gute Gelegenheit, bei der eine Fachgesellschaft zeigen kann, wie sie sich den künftigen Herausforderungen stellt und welche Anpassungen für die nächsten sieben Jahre geplant sind.

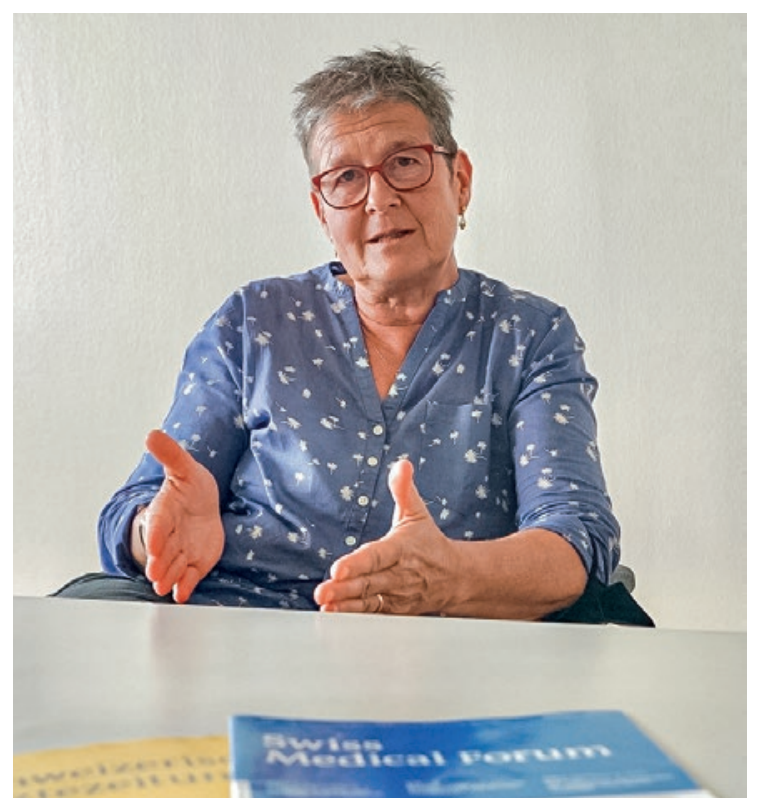

Monika Brodmann: « Es braucht flankierend einen Kulturwandel aller beteiligten Player auf sämtlichen Stufen.» 


\section{Zur Person}

PD Dr. med. et MME Monika Brodmann Maeder studierte in Basel Medizin. Als Notfall- und Gebirgsmedizinerin arbeitete die Interlakerin mehr als zehn Jahre auf den Gebirgsbasen der Luftrettungsorganisation Rega und war während dreier Jahre für die Weiterbildung aller Rega-Helikopterteams in der Schweiz verantwortlich. Monika Brodmann absolvierte einen Masterstudiengang in Medical Education (MME) der Universität Bern. Dabei setzte sich die Ärztin intensiv mit dem Thema Interprofessionelle Weiterbildung auseinander, was ihr später erlaubte, ihre Expertise in diesem Bereich im Berufsalltag einfliessen zu lassen. Die 58-jährige Fachärztin für Allgemeine Innere Medizin mit Fähigkeitsausweisen in präklinischer und klinischer Notfallmedizin war bis vor Kurzem Leitende Ärztin am Universitären Notfallzentrum des Inselspitals Bern. Im Jahr 2020 habilitierte sie an der Universität Bern. Am Institut für Alpine Notfallmedizin in Bozen (I) ist Brodmann noch kurze Zeit als Senior Researcher tätig.

Ich habe den Eindruck, dass dank der offenen Kommunikation zwischen BAG und SIWF die Basis für eine effiziente Umsetzung geschaffen werden konnte.

\section{Langfristig wird auch ein Kontinuum zwischen Aus- und Weiterbildung angestrebt. Wie wirkt sich dies auf die Laufbahn künftiger Ärztinnen und Ärzte aus?}

Heute absolvieren die Studierenden ein Medizinstudium, schliessen dieses ab und dann beginnt ein neues Kapitel, bei dem sie wieder bei null beginnen. Dies muss sich ändern. Ziel muss eine kontinuierlich steigende Lernkurve vom Studienbeginn bis zum Abschluss der Weiterbildung mit möglichst gut überwindbaren Stufen sein. Um diese Kontinuität zu erreichen, braucht es jedoch einen engen Austausch zwischen den Fakultäten und den Weiterbildungsverantwortlichen.

\section{Sie haben nun einige Ziele im Weiterbildungsbereich} erläutert. Wie sieht deren zeitliche Umsetzung aus? Das Projekt kompetenzbasierte ärztliche Weiterbildung ist auf rund zehn Jahre ausgelegt. Dann sollte die CBME in allen Weiterbildungsprogrammen implementiert und beim Grossteil der Weiterbildungsstätten Fortschritte bei der Umsetzung der kompetenzorientierung sichtbar sein. Und zu guter Letzt müssten alle Weiterzubildenden die Möglichkeit haben, ihre EPAs zu dokumentieren, um einen Facharzttitel erwerben zu können. Als Kontrollpunkte für das Fortschreiten dieser Prozesse bieten sich die Akkreditierungszeitpunkte des SIWF durch den Bundesrat an. Die nächste Akkreditierung findet im Jahr 2025 statt. Dann wird sichtbar, wie weit die einzelnen Fachgesellschaften im Bereich CBME sind und welches ihre Pläne sind. Wenn unser Prüfbericht vom Bundesrat akzeptiert wird, ist ein erster wichtiger Schritt geschafft. Bei der über- nächsten Akkreditierung im Jahr 2032 lässt sich dann bestimmen, wie weit wir im Bereich der CBME gekommen sind. Der Zeitplan ist ehrgeizig, aber wir müssen Schwung in die Implementierung der CBME bringen, sonst versandet sie.

\section{Lassen Sie uns zum Schluss noch kurz über die Fortbildung sprechen. Was ist in diesem Bereich geplant?}

Da gibt es zwei grössere Handlungsfelder. Das erste ist der Ausbau der e-Fortbildungsplattform, da diese ein wichtiges Instrument der ärztlichen Fortbildung ist. Diese wird im kommenden Jahr mit einem zentralen Veranstaltungskalender und der Möglichkeit, die einzelnen Veranstaltungen mittels QR-Code per Mausklick ins persönliche Fortbildungsprotokoll aufzunehmen, ausgebaut. Ziel ist, dass alle fortbildungspflichtigen

Der Zeitplan ist ehrgeizig, aber wir müssen Schwung in die Implementierung der CBME bringen, sonst versandet sie.

Ärztinnen und Ärzte ein Fortbildungsdiplom erwerben. Die Ärzteschaft muss sich gegenüber den Behörden und der Öffentlichkeit über die gesetzlich geforderte Fortbildungspflicht ausweisen können. Zurzeit haben erst ca. $40 \%$ der Ärztinnen und Ärzte ein Fortbildungsdiplom.

\section{Und zweitens?}

Ganz aktuell läuft eine Vernehmlassung in den Fachgesellschaften zum Bericht «Ärztliche Fortbildung in der Schweiz: Standortbestimmung und Perspektiven»" Dieser Bericht, den das SIWF im Auftrag des BAG letztes Jahr herausgegeben hat, beschreibt den aktuellen Stand der ärztlichen Fortbildung und Schritte, die zu einer weiteren Verbesserung deren Qualität führen könnten. Danach eruieren wir gemeinsam, welche Konsequenzen wir aus dem Dokument ziehen können, um im Winterhalbjahr möglichst konkrete und praktikable Empfehlungen aussprechen zu können. Schlussendlich müssen wir Antworten auf Fragen, wie sich Ärztinnen und Ärzte künftig fortbilden möchten, wie wir ihnen die Fortbildungen vereinfachen können und welche Inhalte dabei wichtig sind, finden.

\section{Bildnachweis}

Matthias Scholer

Literatur

1 https://www.siwf.ch/files/pdf25/ber_mandat_fb.pdf 